Review

\title{
Relationship between Progression-free Survival and Overall Survival in Randomized Clinical Trials of Targeted and Biologic Agents in Oncology
}

\author{
Lisa M Hess ${ }^{1}$, Alan Brnabic², Oksana Mason'1, Pablo Lee ${ }^{1}$, Scott Barker ${ }^{1}$ \\ 1. Eli Lilly and Company, Indianapolis USA \\ 2. Eli Lilly and Company, Sydney Australia \\ $\triangle$ Corresponding author: Lisa M Hess, PhD, Eli Lilly and Company, Indianapolis IN 46285, (317) 908-1872. Hess_lisa_m@lilly.com \\ (c) Ivyspring International Publisher. This is an open access article distributed under the terms of the Creative Commons Attribution (CC BY-NC) license \\ (https://creativecommons.org/licenses/by-nc/4.0/). See http://ivyspring.com/terms for full terms and conditions.
}

Received: 2018.12.12; Accepted: 2019.05.15; Published: 2019.06.09

\begin{abstract}
Introduction: With a gap in a full understanding of the mechanisms by which survival is extended for patients with cancer who are treated with novel biologic and targeted agents, there is the risk that discordant progression-free and overall survival outcomes are observed due to poor clinical trial design or biases in the interpretation of data. This study was designed to examine the role of study quality and design on the outcomes observed with biologic and targeted agents.

Methods: A review of studies in clinicaltrials.gov supplemented with a literature review in OVID Medline was conducted to identify all randomized trials of a biologic/targeted agent versus a non-biologic/targeted comparator in oncology that report both median overall and progression-free survival outcomes. Details of the study, design, population, drugs, and outcomes were extracted. Study quality was evaluated using the PEDro scale. Data were summarized using SPSS 22.0.0.0.

Results: A total of 192 unique studies of 206 pairwise comparisons between a biologic/targeted and comparator were identified. The average absolute magnitude of post-progression survival (difference between OS and PFS) was 9.7 months for biologic/targeted therapy and 9.8 for the comparator. A total of 64 comparisons (31.1\%) showed an increase in OS and decrease in PFS, or vice versa, and 25 (12.1\%) showed a magnitude of more than 4 months difference between the delta of OS and delta of PFS between the biologic/targeted and comparator arms. Average study quality was high overall (7.7/10), and was comparable for studies with directional differences $(7.2 / 10)$ as well as for those with the greatest magnitude in post-progression survival (7.4/10).

Conclusion: This review and analysis specifically examined small PFS benefit with large OS benefit as well as small OS benefit with large PFS benefit, including differences in direction of PFS and OS outcomes. No evidence was identified that these are the result of poor study design, but may rather be due to the mechanism of action, specific disease, and population under study. Further work is needed to understand the mechanism of action of novel biologic/targeted agents to better understand their interaction with the tumor microenvironment.
\end{abstract}

Key words: biologic therapy, overall survival, post-progression survival, disease progression, oncology

\section{Introduction}

A requirement of new drug approvals in oncology by the U.S. Food and Drug Administration (FDA) and other regulatory bodies is that the drug shows direct clinical benefit or indirect clinical benefit through a surrogate endpoint [1]. Progression-free survival (PFS), the time from treatment initiation until disease progression or worsening, may be used as a direct or surrogate measure of clinical benefit for drug approvals, depending on the disease and response observed, while overall survival (OS), the duration of patient survival from the time of treatment initiation, is a universally-accepted direct measure of clinical benefit.

While improvements in OS clearly demonstrate clinical benefits that are meaningful to patients, PFS, depending on the magnitude, may have high value as 
well. By design, PFS and OS will be related, as OS is comprised of PFS plus post-progression survival. However, the relationship between PFS and OS is not always straightforward. In some diseases, such as advanced colorectal and ovarian cancers, there is a strong correlation between the two outcomes, while in others, like sarcoma, advanced breast cancer, prostate cancer and NSCLC, the relationship is less clear $[2,3]$. The relationship between PFS and OS becomes more complex in the context of biologic and targeted therapies [3,4]. Some of the recent novel targeted and immunologic agents have demonstrated relatively low PFS improvements, but dramatic improvements in OS (long periods of post-progression survival) [5, 6].

The development of novel targeted or biologic therapies (e.g. therapies that target specific mechanisms in a cancer cell, such as immune checkpoint inhibitors, or those that are derived from living organisms, such as antibodies and vaccines) has been of major interest in the field of oncology. As our understanding of the mechanisms of carcinogenesis has evolved, there has been a growth in the development of treatments targeting the tumor microenvironment and immune response. The pathway for checkpoint inhibition supporting the observed outcomes with PD-1 and PDL- 1 inhibitors is relatively well characterized [7]; however, the exact mechanism by which some novel agents affect long-term survival, such those that act on platelet-derived growth factor receptor-alpha (PDGFRa) inhibition, remains elusive [8].

With the development of targeted biologic therapies, consideration must be given to the assessment of disease response using RECIST Criteria [9]. This is particularly challenging, given the various phenotypes and kinetics of tumor responses with targeted therapies. The development of novel imaging techniques to assess the micro-structural properties, as opposed to size-based parameters, as well as the reconsideration of timing assessment criteria, will be paramount in the assessment of these treatment-related changes. Tumor response criteria should take into account the mechanism of action of treatment, biological pathways expressed as well as tumor type and pathogenicity. Although tumor response rate, as measured through RECIST, will remain important, the meaningful benefit has thus far been with prolonged overall survival [4]. The combination of a strong clinical background and thorough understanding of the mechanism of action at work is crucial to the interpretation of the increased survival rates seen with novel targeted therapies.

With a gap in a full understanding of the mechanisms by which survival is extended, there is the risk that discordant response and survival outcomes are observed due to poor clinical trial design or biases in the interpretation of data. For example, design, conduct, analysis and publication flaws have been identified that can bias research findings $[10,11]$. Design flaws may include: failure to conceal allocation, resulting in the subjective selection of patients perceived to be most appropriate for the treatment assignment; non-blinded designs, while may be the only solution in oncology if agents are being compared that have different infusion schedules, treatment schedules or toxicity profiles; study personnel may evaluate or remove participants from treatment differentially between arms if there is knowledge of which treatment is being administered; and imbalance in randomized groups that may be in part due to failure to stratify on key prognostic factors. Biases in the analysis and publication of trial results can be due to selective reporting of outcomes, analyses that are not conducted as intent-to-treat (e.g. exclusion of some randomized participants), and gaps in reported data (e.g. point estimates without measures of variability) [12]. In some ways, PFS is an outcome with some limitations, as it can only be measured at the time of a scheduled imaging scan or other assessment (e.g. leading to periodic intervals at which events are measured regardless of when it actually occurred), whereas OS can be measured to the exact day of the event.

As the number and complexity of new targeted and biologic agents grow, it becomes more complex to interpret and rely on the scientific evidence when the typical patterns of survival and response are not observed, as had been more common with cytotoxic agents. To address this issue, this study was designed to explore the relationship between PFS and OS observed in randomized trials of biologic and targeted agents. The primary purpose of this study was to evaluate the results of clinical trials reporting PFS and OS outcomes to examine situations in which these outcomes may differ (one increases, the other decreases) or where there may be large periods of post-progression survival differences. Observed differences of direction and magnitude were further evaluated in the context of study quality to identify the risk of study design flaws that may influence measured survival outcomes.

\section{Methods}

A clinical trial review was conducted within the U.S. National Library of Medicine's clinical trials registry (clinicaltrials.gov) supplemented by a search of the published literature. The clinicaltrials.gov search was conducted using i2E (Linguamatics Inc), a natural language processing text mining software. 
The i2E query for clinicaltrials.gov included the terms Phase 2 or 3, biologic or targeted agents, cancer terms, and survival data without restriction on the year of the study or results. This was supplemented with a literature search in Ovid using the MEDLINE and pre-MEDLINE databases using similar terms as the i2E search for publications since 2014 (to capture any data that may not yet have been entered into clinicaltrials.gov). The search strategies were executed in April 2018 (complete search strategies are shown in the Supplemental Materials).

Eligible studies were limited to randomized trials that had a biologic or targeted agent in at least one, but not all, treatment arms. The minimum data reporting requirements included median PFS and OS. All cancer types and populations were eligible for inclusion; however, each trial was required to study a specific tumor type, so that trials of multiple tumor types were excluded. Eligible studies were published in English and enrolled adult cancer patients. Two independent reviewers evaluated all potential studies for eligibility. The final set of eligible studies underwent extensive data extraction to obtain study details according to PICO (population, intervention, control, outcome) criteria. Population variables included sample size, demographics and disease site. Intervention and control variables included the agents and regimens used, dose, duration and line of therapy. Outcomes included PFS, OS and tumor response. Additional variable collected included study quality using the Physiotherapy Evidence Database (PEDro) scale. This scale reports study quality across 11 items, with a total quality score ranging from 0-10 (the first item is related to external generalizability and is not included in the total score); scores above 6 generally represent good study quality, and scores below that level are poor study quality. In addition to the PEDro scale, imbalances in post-study therapies were recorded, as these may influence subsequent OS outcomes in oncology.

Studies were summarized descriptively. The difference in median months of OS and PFS (delta OS and delta PFS) was calculated between biologic/targeted and comparator (non-biologic and non-targeted) therapy. For trials with multiple treatment arms, these were calculated for each biologic/targeted therapy versus the comparator. Negative differences indicate that the comparator performed better than the biologic/targeted therapy, whereas positive differences indicate that the biologic/targeted therapy performed better.

Post-progression survival (PPS) is the time the patient is alive following disease progression (OS = PFS + PPS). To evaluate PPS, median PFS was subtracted from the median OS for each treatment arm, and the difference in PPS for the comparator from that of the biologic/targeted therapy was summarized (delta PPS $=$ PPS $_{\text {biologic }}-$ PPS $_{\text {comparator }}$ ). The difference in OS and PFS were also calculated (delta $\mathrm{OS}=\mathrm{OS}_{\text {biologic }}-\mathrm{OS}_{\text {comparator; }}$ delta PFS $=\mathrm{PFS}_{\text {biologic }}-$ PFS $_{\text {comparator, }}$ respectively). To explore the difference in PPS between arms, the Delta PPS was calculated (deltaOS - delta PFS). Studies with differential outcomes (e.g. Delta PFS $>0$ months and delta OS $\leq 0$ months, or delta PFS $\leq 0$ months and delta OS $>0$ months) were summarized. Biologic/targeted therapies with large delta OS but small delta PFS as well as those with large delta PFS but small delta OS were also summarized. Absolute ratios between delta PFS and delta OS were explored as the primary outcome of this study but not pursued as an endpoint, as large ratios may result from small numerical differences (e.g. 3 days of PFS benefit and 1 months of OS benefit result in a 30:1 ratio, whereas a 2 month PFS and a 10 month OS benefit is only a 5:1 ratio). Due to the lack of direct clinical relevance of ratios, these were ultimately not included in this study.

SPSS 22.0.0.0. (IBM Corporation) and Excel 2016 (Microsoft, Inc.) were used to conduct analyses and to generate the summary figures.

\section{Results}

After applying eligibility criteria and excluding duplicates, a total of 24 clinicaltrials.gov records and 168 publications (representing 192 unique studies and 206 pairwise comparisons between biologic/targeted versus comparator) met eligibility criteria and were included in this analysis (the full list of included publications is provided in the Supplemental Materials). The number of eligible publications of trials comparing chemotherapy agents by year of publication are summarized in Figure 1. The most common tumor sites included lung $(\mathrm{n}=60,31.3 \%)$, breast $(\mathrm{n}=24,12.5 \%)$, colorectal $(\mathrm{n}=18,9.4 \%)$, gastric/esophageal $(n=17,8.9 \%)$, pancreatic $(n=13$, $6.8 \%)$, gynecologic $(n=8,4.2 \%)$ and glioblastoma $(n=8$, $4.2 \%)$. Most eligible studies investigated first-line therapy ( $\mathrm{n}=87,45.3 \%) ; 53(27.6 \%)$ were second-line trials, $9(4.7 \%)$ investigated maintenance therapy, 2 $(1.0 \%)$ studied neoadjuvant or adjuvant therapy and the remaining were not limited to a single line of therapy.

\section{Differences in PFS and OS}

There were 206 pairwise comparisons between biologic/targeted and comparator therapy across all included studies due to the inclusion of multiple-arm trials. The distribution of delta in OS and PFS of these comparisons is presented in in Figure 2 (excluding dual publications of the same study), sorted by 
magnitude in OS improvement. Values close to zero indicate similar magnitude of improvement between the biologic/targeted and comparator (non-biologic/targeted) therapy. Higher values indicate greater improvement associated with the biologic/targeted therapy, and lower values indicate greater improvement associated with the comparator therapy. Overall survival associated with the biologic/targeted therapy ranged from 9.8 months lower than the comparator to a gain of 25.4 months versus the comparator. PFS associated with the biologic/targeted therapy ranged from 8.2 months lower than the comparator to 14.9 months more than the comparator. Biologic/targeted therapies improved both OS and PFS an average of 1.2 months (SD: $\quad 3.8$ and 2.5, respectively) versus non-biologic/targeted therapy.

The absolute magnitude of PPS for patients treated with biologic/targeted therapy was an average of 9.7 months (standard deviation, $\mathrm{SD}=7.4$ ), and the absolute magnitude of PPS for the non-biologic/targeted comparator was 9.8 months $(\mathrm{SD}=7.6)$. Figure 3 presents the delta PPS across all comparisons included in this study.

\section{Direction of survival outcomes}

The direction of OS and PFS findings was not consistent across all studies. While there were 33 $(16.0 \%)$ comparisons in which both OS and PFS were lower for patients treated in the biologic/targeted arm than the comparator, and 104 (50.5\%) in which OS and PFS were both higher with the biologic/targeted therapy; 39 (18.9\%) showed improvement in PFS but none in OS, and $25(12.1 \%)$ showed improvement in OS but none in PFS. The studies demonstrating differential results between PFS and OS are summarized in Tables 1 and 2. Among the comparisons that demonstrated inconsistent results between PFS and OS, the magnitude of inconsistency was relatively low. Among the 39 showing improvement in PFS but not OS, the average PFS benefit was 1.5 months (standard deviation [SD] =

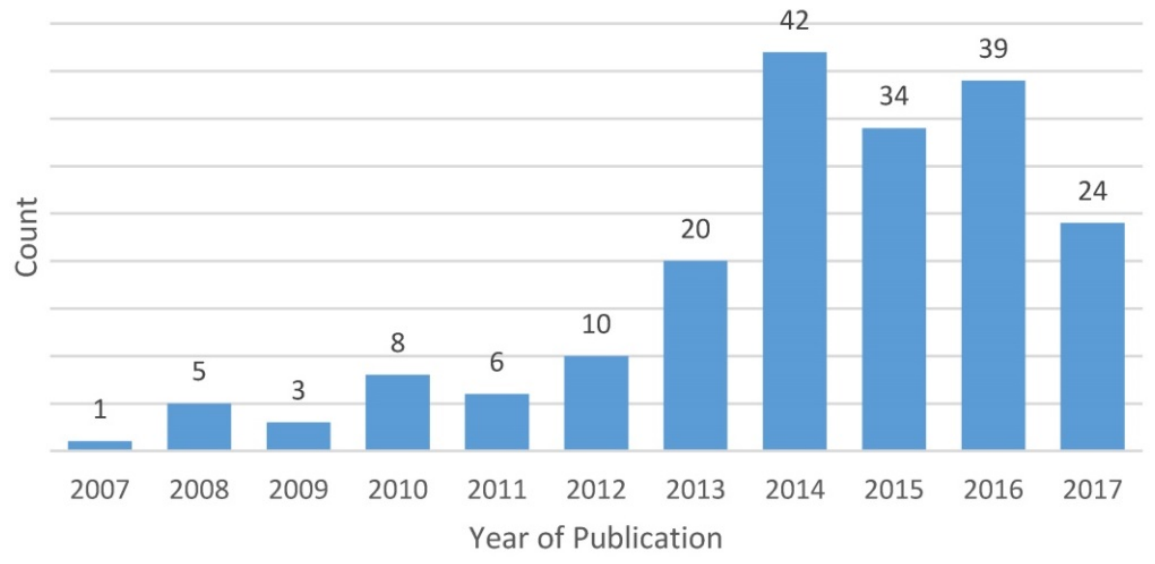

Figure 1. Year of publication of eligible studies $(n=192)$

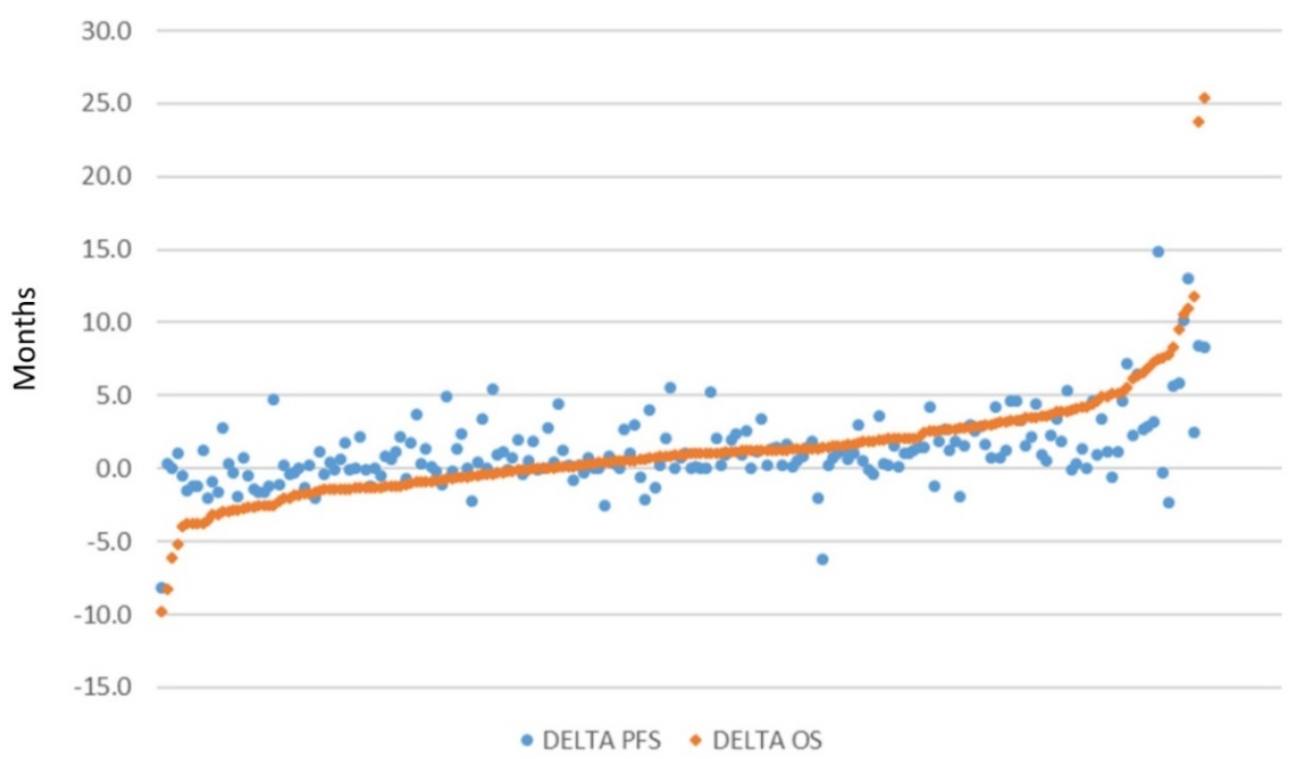

Figure 2. Scatterplot of difference in median months of overall survival (OS) and median progression-free survival (PFS), respectively, between treatment arms ( $\mathrm{n}=206$ ) 


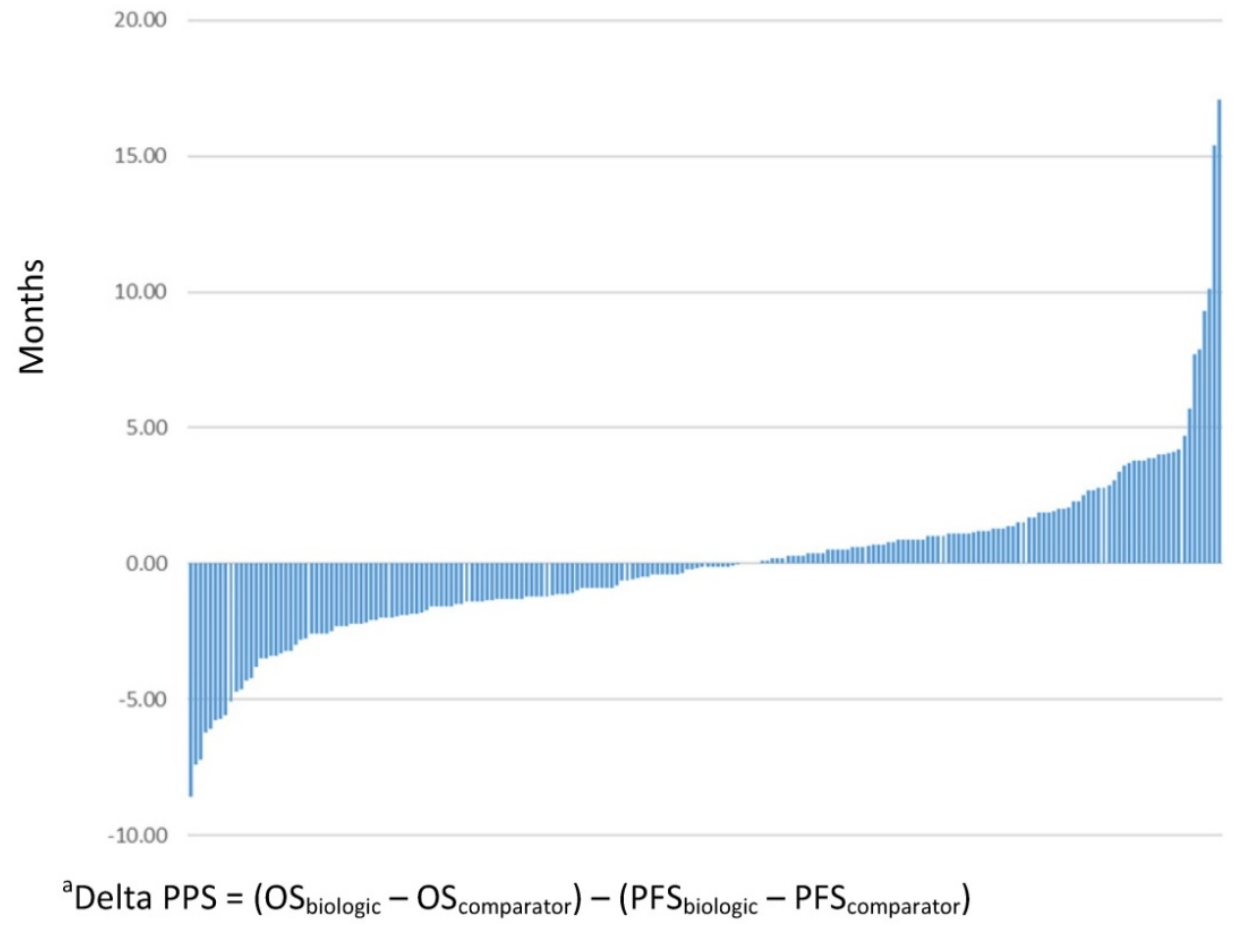

Figure 3. Delta post-progression survival (PPS) between biologic/targeted and non-biologic/targeted comparatora $(\mathrm{N}=206)$

1.4), and the average decline in OS was -1.4 months $(\mathrm{SD}=1.6)$. Among the 25 comparisons with improvements in OS but not PFS, the average benefit in OS was 2.0 months (SD = 2.2), and the average decline in PFS was -0.9 months $(\mathrm{SD}=1.4)$.

\section{Magnitude of survival difference}

Comparisons with at least a 4-month difference in delta PPS $(n=25)$ are presented in Table 3. Of these, 16 showed OS benefits biologic/targeted therapy, and 9 showed no benefit in OS for the patients treated with biologic/targeted therapy. Studies of biologic/targeted therapies that improved OS investigated regimens that included pembrolizumab, nivolumab, gefitinib and erlotinib in NSCLC, trastuzumab in breast cancer, lapatinib in breast cancer and SCCHN, rituximab and bortezomib in mantle cell lymphoma, bevacizumab in glioblastoma, vandetanib in SCLC, and olaratumab in soft tissue sarcoma, and MK-2206, a novel AKT inhibitor. Other than MK-2206, which is still in development, and vendetanib, which was associated with increased toxicity [13], each of these other agents had FDA approval and/or NCCN guideline placement for use in these diseases. In February 2019, olaratumab was removed from NCCN clinical practice guidelines following the results of a phase III clinical trial, which found no difference in the primary endpoint of overall survival between study arms [14].

\section{Study quality}

The average PeDRO score of included studies was 7.7 (SD: 1.8). Quality scores ranged from 3 to 10. Only 24 (12.6\%) were considered low-quality studies, and 15 of these $(62.5 \%)$ were not published (e.g. clinicaltrials.gov record only). Studies with differential PFS and OS outcomes had an average score of 7.2 (SD: 1.7). Studies with the largest differences in OS-PFS between biologic/targeted and comparator arms had an average quality score of 7.4 (SD: 1.7). Most studies (127, 66.5\%) did not report post-study therapies received. However, among the $64(33.5 \%)$ that did report these data, 41 (64.1\%) showed differences in subsequent treatments received, and $23(35.9 \%)$ reported similarities in post-study therapies. Of those 23 with similar post-study therapies, three $(13.0 \%)$ showed differential OS/PFS direction of benefit,[15-17] and 2 $(8.7 \%)$ showed large magnitude in the difference between OS and PFS outcomes.[5, 15] Of studies with imbalanced post-study therapies, 19 (46.3\%) found directional differences in OS/PFS and $10(24.3 \%)$ showed large differences in OS/PFS, and $12(29.3 \%)$ found a no benefit in OS with the biologic/targeted therapy. Among all studies with a benefit in OS, only $49(36.8 \%)$ reported post study therapies; of those, 29 (59.2\%) reported imbalance. Among all studies with no benefit in OS, $15(20.5 \%)$ reported post-study therapies; of those, $12(80 \%)$ had imbalance in post-study therapies. The average study quality of studies reporting benefits in OS was 7.8/10 $(\mathrm{SD}=1.8)$ and for those with no OS benefit the average study quality was $7.4 / 10(\mathrm{SD}=1.7)$. 
Table 1. Comparisons showing improvement (>0 months) in progression-free survival (PFS) and no improvement ( $\leq 0$ months) in overall survival $(n=39)$

\begin{tabular}{|c|c|c|c|c|c|c|c|}
\hline Reference & Disease site & Line of therapy & Treatment comparison & $\mathbf{N}$ & $\begin{array}{l}\text { Median OS } \\
\text { (months) }\end{array}$ & $\begin{array}{l}\text { Median PFS } \\
\text { (months) }\end{array}$ & $\begin{array}{l}\text { Study } \\
\text { Quality }\end{array}$ \\
\hline \multirow[t]{2}{*}{ Beer et al. 2017[18] } & \multirow[t]{2}{*}{ Prostate } & \multirow[t]{2}{*}{1} & ipilumumab & 400 & 28.7 & 5.6 & \multirow[t]{2}{*}{9} \\
\hline & & & placebo & 202 & 29.7 & 3.8 & \\
\hline \multirow[t]{2}{*}{ Belani et al. 2014[19] } & \multirow[t]{2}{*}{ NSCLC } & \multirow[t]{2}{*}{1} & axitinib + pemetrexed + cisplatin & 58 & 14.7 & 7.9 & \multirow[t]{2}{*}{8} \\
\hline & & & pemetrexed + cisplatin & 57 & 15.9 & 7.1 & \\
\hline \multirow[t]{2}{*}{ Blumenschein et al. 2015[20] } & \multirow[t]{2}{*}{ NSCLC } & \multirow[t]{2}{*}{2} & trametinib & 86 & 8.1 & 2.7 & 6 \\
\hline & & & docetaxel & 43 & 9.9 & 2.6 & \\
\hline Burtness et al. 2016 [21] & Pancreatic & 1 & irinotecan + docetaxel + cetuximab & 45 & 5.3 & 4.5 & 7 \\
\hline & & & irinotecan + docetaxel & 46 & 6.5 & 3.9 & \\
\hline Chauffert et al. 2014[22] & Glioblastoma & Neoadjuvant/ & bevacizumb + irinotecan & 60 & 11.1 & 7.1 & 7 \\
\hline & & adjuvant & temozolomide & 60 & 11.1 & 5.2 & \\
\hline Doebele et al. 2015[23] & NSCLC & 1 & ramucirumab + gemcitabine + cisplatin & 71 & 9.8 & 5.6 & 6 \\
\hline & & & gemcitabine + cisplatin & 69 & 11.8 & 5.4 & \\
\hline Evans et al. 2017[24] & Pancreatic & 1 & dasatinib + gemcitabine & 100 & 12.33 & 5.49 & 9 \\
\hline & & & placebo + gemcitabine & 102 & 12.93 & 5.46 & \\
\hline Gilbert et al. 2014[25] & Glioblastoma & 1 & bevacizumab & 320 & 15.7 & 10.7 & 9 \\
\hline & & & placebo & 317 & 16.1 & 7.3 & \\
\hline Gridelli et al. 2014[26] & NSCLC & 1 & vandetanib + gemcitabine & 61 & 8.6 & 6.0 & 9 \\
\hline & & & placebo + gemcitabine & 63 & 10.0 & 5.6 & \\
\hline Hegewisch-Becker, et al. & Colorectal & Maintenance & fluoropyrimidine + bevacizumab & 158 & 20.2 & 6.3 & 7 \\
\hline $2015[15]$ & & & no maintenance & 158 & 23.1 & 3.5 & \\
\hline Hegewisch-Becker et al. & Colorectal & Maintenance & bevacizumab & 156 & 21.9 & 4.6 & 7 \\
\hline $2015[15]$ & & & no maintenance & 158 & 23.1 & 3.5 & \\
\hline Herrlinger et al. 2016[27] & Glioblastoma & 1 & bevacizumb + irinotecan & 122 & 16.6 & 9.7 & 7 \\
\hline & & & temozolomide & 60 & 17.5 & 6.0 & \\
\hline Kim et al. 2013[28] & NSCLC & 2 & cetuximab + pemetrexed & 301 & 6.9 & 2.9 & 7 \\
\hline & & & pemetrexed & 304 & 7.8 & 2.8 & \\
\hline Leone et al. 2016[29] & Biliary tract & 1 & gemcitabine + oxaliplatin + panitumumab & 45 & 9.9 & 5.3 & 7 \\
\hline & & & gemcitabine + oxaliplatin & 44 & 10.2 & 4.4 & \\
\hline Li et al. 2014[30] & NSCLC & 2 & erlotinib & 61 & 11.7 & 4.1 & 6 \\
\hline & & & pemetrexed & 62 & 13.4 & 3.9 & \\
\hline Malka et al. 2014[31] & Biliary tract & 1 & gemcitabine + oxaliplatin + cetuximab & 76 & 11.0 & 6.1 & 6 \\
\hline & & & gemcitabine + oxaliplatin & 74 & 12.4 & 5.5 & \\
\hline Martin et al. 2015[32] & Breast & 1 & letrozole or fulvestrant + bevacizumab & 191 & 52.1 & 19.3 & 6 \\
\hline & & & letrozole or fulvestrant & 189 & 52.8 & 14.4 & \\
\hline Masi et al. 2015[33] & Colorectal & 2 & FOLFIRI or FOLFOX6 + bevacizumab & 92 & 14.1 & 6.8 & 7 \\
\hline & & & FOLFIRI or FOLFOX6 & 92 & 15.5 & 5.0 & \\
\hline McDermott et al. 2008[34] & Melanoma & 1 & sorafenib + dacarbazine & 51 & 10.5 & 4.9 & 9 \\
\hline & & & placebo + dacarbazine & 50 & 11.8 & 2.7 & \\
\hline Middleton et al. 2017[35] & Pancreatic & 1 & vandetanib + gemcitabine & 72 & 8.8 & 8.0 & 9 \\
\hline & & & placebo + gemcitabine & 70 & 9.0 & 6.1 & \\
\hline Miller et al. 2012[36] & NSCLC & $2+$ & atafanib & 390 & 10.8 & 3.3 & 9 \\
\hline & & & placebo & 195 & 12.0 & 1.1 & \\
\hline Moore et al. 2016[37] & Colorectal & 2 & ramucirumab + modified FOLFOX6 & 52 & 9.6 & 4.9 & 5 \\
\hline & & & modified FOLFOX6 & 54 & 12.3 & 4.2 & \\
\hline NCT00110019[38] & Melanoma & 1 & paclitaxel + carboplatin + sorafenib & 410 & 11.1 & 4.9 & 8 \\
\hline & & & paclitaxel + carboplatin + placebo & 413 & 11.3 & 4.2 & \\
\hline NCT00393939[39] & Breast & 1 & sunitinib + docetaxel & 296 & 26.0 & 8.6 & 5 \\
\hline & & & docetaxel & 297 & 28.9 & 8.3 & \\
\hline NCT00403403[40] & SCLC & 1 & $\begin{array}{l}\text { cisplatin or carboplatin }+ \text { etoposide }+ \\
\text { bevacizumab }\end{array}$ & 52 & 9.4 & 5.5 & 7 \\
\hline & & & $\begin{array}{l}\text { cisplatin or carboplatin }+ \text { etoposide }+ \\
\text { placebo }\end{array}$ & 50 & 10.9 & 4.4 & \\
\hline NCT00459043[41] & $\mathrm{SCCHN}$ & Multiple & vandetanib + docetaxel & 15 & 5.6 & 2.1 & 3 \\
\hline & & & docetaxel & 15 & 6.2 & 0.7 & \\
\hline NCT00777179[42] & NSCLC & Maintenance & vandetanib & 75 & 15.6 & 2.7 & 7 \\
\hline & & & placebo & 42 & 20.8 & 1.7 & \\
\hline NCT01234337[43] & Breast & $1+$ & sorafenib + capecitabine & 266 & 18.9 & 5.5 & 10 \\
\hline & & & placebo + capecitabine & 271 & 20.3 & 5.4 & \\
\hline Oza et al. 2015[44] & Ovarian & 1 & carboplatin + paclitaxel + bevacizumab & 764 & 58.0 & 19.9 & 8 \\
\hline & & & carboplatin + paclitaxel & 764 & 58.6 & 17.5 & \\
\hline Paz-Ares et al. 2012[45] & NSCLC & 1 & gemcitabine + cisplatin + sorafenib & 452 & 12.4 & 6.0 & 10 \\
\hline & & & gemcitabine + cisplatin + placebo & 452 & 12.5 & 5.5 & \\
\hline Ramlau et al. 2012[46] & NSCLC & 2 & aflibercept + docetaxel & 456 & 10.1 & 5.2 & 10 \\
\hline & & & placebo + docetaxel & 457 & 10.4 & 4.1 & \\
\hline Schuler et al. 2016[47] & NSCLC & $2+$ & afatinib + paclitaxel & 134 & 12.2 & 5.6 & 6 \\
\hline & & & chemotherapy & 68 & 12.2 & 2.8 & \\
\hline Shaw et al. 2013[48] & NSCLC & 2 & crizotinib & 173 & 20.3 & 7.7 & 8 \\
\hline & & & pemetrexed or docetaxel & 174 & 22.8 & 3.0 & \\
\hline Shen et al. 2015[49] & Gastric & 1 & capecitabine + cisplatin + bevacizumab & 102 & 10.5 & 6.3 & 10 \\
\hline
\end{tabular}




\begin{tabular}{|c|c|c|c|c|c|c|c|}
\hline Reference & Disease site & Line of therapy & Treatment comparison & $\mathbf{N}$ & $\begin{array}{l}\text { Median OS } \\
\text { (months) }\end{array}$ & $\begin{array}{l}\text { Median PFS } \\
\text { (months) }\end{array}$ & $\begin{array}{l}\text { Study } \\
\text { Quality }\end{array}$ \\
\hline & & & capecitabine + cisplatin + placebo & 100 & 11.4 & 6.0 & \\
\hline \multirow[t]{2}{*}{ Tabernero et al. 2013[17] } & Colorectal & 1 & modified FOLFOX6 + sorafenib & 97 & 17.6 & 9.1 & 9 \\
\hline & & & modified FOLFOX6 + placebo & 101 & 18.1 & 8.7 & \\
\hline \multirow[t]{2}{*}{ Vergote et al. 2014[50] } & Ovarian & Maintenance & erlotinib & 420 & 50.8 & 12.7 & 7 \\
\hline & & & no treatment & 415 & 59.1 & 12.4 & \\
\hline \multirow[t]{2}{*}{ Vincent et al. 2017[51] } & Colorectal & 1 & erlotinib + capecitabine & 42 & 12.4 & 9.2 & 7 \\
\hline & & & capecitabine & 40 & 16.2 & 7.9 & \\
\hline \multirow[t]{2}{*}{ Wirth et al. 2016[52] } & SCCHN & 1 & docetaxel + cisplatin + panitumumab & 56 & 12.9 & 6.9 & 5 \\
\hline & & & docetaxel + cisplatin & 57 & 13.8 & 5.5 & \\
\hline \multirow[t]{2}{*}{ Wu et al. 2014[53] } & NSCLC & 1 & afatinib & 242 & 23.1 & 11.0 & 7 \\
\hline & & & gemcitabine + cisplatin & 122 & 23.5 & 5.6 & \\
\hline
\end{tabular}

NSCLC=non-small cell lung cancer; SCLC=small cell lung cancer; SCCHN=squamous cell carcinoma of the head and neck; FOLFIRI=fluorouracil + irinotecan;

FOLFOX=fluorouracil + oxaliplatin

Table 2. Comparisons showing improvement (>0 months) in overall survival and no improvement ( $\leq 0$ months) in progression-free survival $(n=25)$

\begin{tabular}{|c|c|c|c|c|c|c|c|}
\hline Reference & Disease site & Line of therapy & Treatment comparison & $\mathbf{N}$ & Median OS (months) & Median PFS (months) & Study Quality \\
\hline \multirow[t]{2}{*}{ Allen et al. 2014[54] } & SCLC & 2 & topotecan + ziv-aflibercept & 55 & 4.6 & 1.4 & 8 \\
\hline & & & topotecan & 51 & 4.2 & 1.4 & \\
\hline \multirow[t]{2}{*}{ Belani et al. 2016[55] } & SCLC & 1 & cisplatin + etoposide + vismodegib & 52 & 9.8 & 4.4 & 6 \\
\hline & & & cisplatin + etoposide & 48 & 8.8 & 4.4 & \\
\hline \multirow[t]{2}{*}{ Bokemeyer et al. 2009[56] } & Colorectal & 1 & FOLFOX $4+$ cetuximab & 170 & 18.3 & 7.2 & 7 \\
\hline & & & FOLFOX4 & 168 & 18.0 & 7.2 & \\
\hline \multirow[t]{2}{*}{ Borghaei et al. 2015[57] } & NSCLC & 2 & nivolumab & 292 & 12.2 & 2.3 & 6 \\
\hline & & & docetaxel & 290 & 9.4 & 4.2 & \\
\hline \multirow[t]{2}{*}{ Buikhuisen et al. 2016[58] } & Mesothelioma & 1 & axitinib & 14 & 18.9 & 5.8 & 4 \\
\hline & & & no treatment & 11 & 18.5 & 8.3 & \\
\hline \multirow[t]{2}{*}{ Govindan et al. 2017[59, 60] } & NSCLC & 1 & chemotherapy + ipilimumab & 479 & $10.9^{*}$ & 5.6 & 8 \\
\hline & & & chemotherapy + placebo & 477 & $10.7^{*}$ & 5.6 & \\
\hline \multirow[t]{2}{*}{ Han et al. 2017[61] } & NSCLC & 1 & gefitinib & 41 & 25.8 & 5.7 & 7 \\
\hline & & & pemetrexed + carboplatin & 40 & 24.3 & 11.9 & \\
\hline \multirow[t]{2}{*}{ Herbst et al. 2016[62] } & NSCLC & $2+$ & pembrolizumab $2 \mathrm{mg} / \mathrm{kg}$ & 345 & 10.4 & 3.9 & 7 \\
\hline & & & docetaxel & 343 & 8.5 & 4.0 & \\
\hline \multirow[t]{2}{*}{ Herbst et al. 2016[62] } & NSCLC & $2+$ & pembrolizumab $10 \mathrm{mg} / \mathrm{kg}$ & 346 & 12.7 & 4.0 & 7 \\
\hline & & & docetaxel & 343 & 8.5 & 4.0 & \\
\hline \multirow[t]{2}{*}{ Jonasch et al. 2017[63] } & Renal cell & $2+$ & MK-2206 & 29 & 23.5 & 3.7 & 5 \\
\hline & & & everolimus & 14 & 15.7 & 6.0 & \\
\hline \multirow[t]{2}{*}{ Kawaguchi et al. 2014[16] } & NSCLC & $2+$ & erlotinib & 150 & 14.8 & 2.0 & 7 \\
\hline & & & docetaxel & 151 & 12.2 & 3.2 & \\
\hline \multirow[t]{2}{*}{ Lee et al. 2013[64] } & NSCLC & 2 & erlotinib & 82 & 22.8 & 3.8 & 7 \\
\hline & & & pemetrexed & 80 & 17.7 & 4.4 & \\
\hline \multirow[t]{2}{*}{ Lee et al. 2014[65] } & NSCLC & $2+$ & erlotinib & 40 & 3.4 & 1.6 & 9 \\
\hline & & & placebo & 40 & 2.9 & 1.6 & \\
\hline \multirow[t]{2}{*}{ NCT00069095[66] } & Colorectal & 1 & (FOLFOX4 or XELOX) + bevacizumab & 699 & 18.9 & 8.0 & 7 \\
\hline & & & $($ FOLFOX4 or XELOX) + placebo & 701 & 18.1 & 9.4 & \\
\hline \multirow[t]{2}{*}{ NCT00448279[67] } & Breast & 2 & chemotherapy + trastuzumab & 29 & 26.7 & 9.4 & 4 \\
\hline & & & chemotherapy & 29 & 19.1 & 9.7 & \\
\hline \multirow[t]{2}{*}{ NCT00597116[68] } & Mesothelioma & 2 & vandetanib & 14 & 7.8 & 1.8 & 3 \\
\hline & & & vinorelbine & 11 & 6.4 & 3.8 & \\
\hline \multirow[t]{2}{*}{ NCT00887159[69] } & SCLC & 1 & cisplatin + etoposide + vismodegib & 56 & 9.8 & 4.4 & 5 \\
\hline & & & cisplatin + etoposide & 56 & 8.8 & 4.4 & \\
\hline \multirow[t]{2}{*}{ NCT01585987[70] } & Gastric & 2 & ipilimumab & 57 & 12.7 & 2.7 & 5 \\
\hline & & & best supportive care & 57 & 12.1 & 4.9 & \\
\hline \multirow[t]{2}{*}{ Pirker et al. 2009[71] } & NSCLC & 1 & cetuximab + cisplatin + vinorelbine & 557 & 11.3 & 4.8 & 7 \\
\hline & & & cisplatin + vinorelbine & 568 & 10.1 & 4.8 & \\
\hline \multirow[t]{2}{*}{ Powles et al. 2017[72] } & Bladder & Maintenance & lapatinib & 116 & 12.6 & 4.5 & 9 \\
\hline & & & placebo & 116 & 12.0 & 5.1 & \\
\hline Propper et al. 2014[73] & Pancreatic & 2 & erlotinib & 104 & 4.0 & 1.4 & 9 \\
\hline & & & placebo & 103 & 3.1 & 1.4 & \\
\hline Sanborn et al. 2017[13] & SCLC & 1 & platinum + etoposide + vandetanib & 34 & 13.2 & 5.6 & 8 \\
\hline & & & platinum + etoposide + placebo & 33 & 9.2 & 5.7 & \\
\hline Scagliotti et al. 2010[74] & NSCLC & 1 & carboplatin + paclitaxel + sorafenib & 464 & 10.7 & 4.6 & 10 \\
\hline & & & carboplatin + paclitaxel + placebo & 462 & 10.6 & 5.4 & \\
\hline Vilgrain et al. 2017[75] & Hepatocellular & 1 & sorafenib & 222 & 9.9 & 3.7 & 8 \\
\hline & & & radiotherapy & 237 & 8.0 & 4.1 & \\
\hline Yoon et al. 2016[76] & Esophagogastric & 1 & modified FOLFOX6 + ramucirumab & 84 & 11.7 & 6.4 & 10 \\
\hline & & & modified FOLFOX6 + placebo & 84 & 11.5 & 6.7 & \\
\hline
\end{tabular}

NSCLC=non-small cell lung cancer; SCLC $=$ small cell lung cancer; FOLFOX=fluorouracil + oxaliplatin; XELOX= capecitabine + oxaliplatin

*Survival data for the full cohort were not published, these data are available in the clinicaltrials.gov record 
Table 3. Comparisons with the greatest differences (positive or negative) in post-progression survival $(n=25)$

\begin{tabular}{|c|c|c|c|c|c|c|c|}
\hline \multicolumn{8}{|c|}{ Comparisons showing a benefit in OS with the biologic/targeted therapy } \\
\hline Reference & Disease site & $\begin{array}{l}\text { Line of } \\
\text { therapy }\end{array}$ & Comparison (n) & $\begin{array}{l}\text { Median OS } \\
\text { (months) }\end{array}$ & $\begin{array}{l}\text { Median PFS } \\
\text { (months) }\end{array}$ & $\begin{array}{l}\text { Delta PPS } \\
\text { between arms }\end{array}$ & $\begin{array}{l}\text { Study } \\
\text { quality }\end{array}$ \\
\hline \multirow[t]{2}{*}{ Rule et al. 2016[77] } & $\begin{array}{l}\text { Mantle cell } \\
\text { lymphoma }\end{array}$ & 1 & $\begin{array}{l}\text { fludarabine }+ \text { cyclophosphamide }+ \text { rituximab } \\
(\mathrm{n}=186)\end{array}$ & 44.5 & 29.8 & -7.4 & 6 \\
\hline & & & fludarabine + cyclophosphamide $(n=184)$ & 37 & 14.9 & & \\
\hline \multirow[t]{2}{*}{ Wu et al. 2015[77] } & NSCLC & 1 & erlotinib $(n=110)$ & 26.3 & 11.0 & -4.7 & 8 \\
\hline & & & gemcitabine + cisplatin $(n=107)$ & 25.5 & 5.5 & & \\
\hline \multirow[t]{2}{*}{ Chinot et al. 2014[78] } & Glioblastoma & 1 & temozolomide + bevacizumab $(n=458)$ & 16.8 & 10.6 & -4.3 & 10 \\
\hline & & & temozolomide + placebo $(n=463)$ & 16.7 & 6.2 & & \\
\hline \multirow{2}{*}{$\begin{array}{l}\text { Schwartzberg et al. } \\
\text { 2010[79] }\end{array}$} & Breast & 1 & lapatinib + letrozole $(n=111)$ & 33.3 & 8.2 & -4.2 & 10 \\
\hline & & & placebo + letrozole $(n=108)$ & 32.3 & 3.0 & & \\
\hline \multirow[t]{2}{*}{ Krop et al. 2017[80] } & Breast & $3+$ & trastuzumab $(n=404)$ & 22.7 & 6.2 & 4.0 & 8 \\
\hline & & & physician choice $(\mathrm{n}=198)$ & 15.8 & 3.3 & & \\
\hline \multirow[t]{2}{*}{ Sanborn et al. 2017[13] } & SCLC & 1 & platinum + etoposide + vandetanib $(n=34)$ & 13.2 & 5.6 & 4.1 & 8 \\
\hline & & & platinum + etoposide + placebo $(n=33)$ & 9.2 & 5.7 & & \\
\hline \multirow[t]{2}{*}{ Guan et al. 2013[81] } & Breast & 1 & lapatinib + paclitaxel $(n=222)$ & 27.8 & 9.7 & 4.1 & 10 \\
\hline & & & placebo + paclitaxel $(n=222)$ & 20.5 & 6.5 & & \\
\hline \multirow[t]{2}{*}{ Herbst et al. 2016[62] } & NSCLC & $2+$ & Pembrolizumab 10mg/kg $(n=346)$ & 12.7 & 4 & 4.2 & 7 \\
\hline & & & docetaxel $(n=343)$ & 8.5 & 4 & & \\
\hline \multirow[t]{2}{*}{ Borghaei et al. 2015[57] } & NSCLC & 2 & nivolumab $(\mathrm{n}=292)$ & 12.2 & 2.3 & 4.7 & 6 \\
\hline & & & docetaxel $(n=290)$ & 9.4 & 4.2 & & \\
\hline \multirow[t]{2}{*}{ Lee et al. 2013[64] } & NSCLC & 2 & erlotinib $(\mathrm{n}=82)$ & 22.8 & 3.8 & 5.7 & 7 \\
\hline & & & pemetrexed $(n=80)$ & 17.7 & 4.4 & & \\
\hline \multirow[t]{2}{*}{ Han et al. 2017[61] } & NSCLC & 1 & gefitinib $(n=41)$ & 25.8 & 5.7 & 7.7 & 7 \\
\hline & & & pemetrexed + carboplatin $(n=40)$ & 24.3 & 11.9 & & \\
\hline \multirow{2}{*}{ NCT00448279[67] } & Breast & 2 & chemotherapy + trastuzumab $(n=29)$ & 26.7 & 9.4 & 7.9 & 4 \\
\hline & & & chemotherapy $(n=29)$ & 19.1 & 9.7 & & \\
\hline \multirow[t]{2}{*}{ Tap et al. 2016[5] } & Sarcoma & $1+$ & olaratumab + doxorubicin $(n=66)$ & 26.5 & 6.6 & 9.3 & 7 \\
\hline & & & doxorubicin $(n=67)$ & 14.7 & 4.1 & & \\
\hline \multirow[t]{2}{*}{ Jonasch et al. 2017[63] } & Renal cell & $2+$ & MK-2206 (n=29) & 23.5 & 3.7 & 10.1 & 5 \\
\hline & & & everolimus ( $n=14)$ & 15.7 & 6.0 & & \\
\hline \multirow[t]{2}{*}{ Furtado et al. 2015[82] } & $\begin{array}{l}\text { Mantle cell } \\
\text { lymphoma }\end{array}$ & 2 & $\begin{array}{l}\text { cyclophosphamide }+ \text { doxorubicin }+ \\
\text { vincristine }+ \text { bortezomib }(n=23)\end{array}$ & 35.6 & 16.5 & 15.4 & 5 \\
\hline & & & $\begin{array}{l}\text { cyclophosphamide }+ \text { doxorubicin }+ \\
\text { vincristine }(n=23)\end{array}$ & 11.8 & 8.1 & & \\
\hline \multirow[t]{2}{*}{ Harrington et al. 2013[83] } & SCCHN & 1 & lapatinib + cisplatin $(n=34)$ & 48.4 & 20.4 & 17.1 & 10 \\
\hline & & & placebo + cisplatin $(n=33)$ & 23.0 & 12.1 & & \\
\hline \multicolumn{8}{|c|}{ Comparisons showing no benefit in OS with the biologic/targeted therapy } \\
\hline \multirow[t]{2}{*}{ Vergote, et al. 2014[50] } & Ovarian & Maintenance & erlotinib $(n=420)$ & 50.8 & 12.7 & -8.6 & 7 \\
\hline & & & no treatment $(n=415)$ & 59.1 & 12.4 & & \\
\hline Shaw et al. 2013[48] & NSCLC & 2 & crizotinib $(\mathrm{n}=173)$ & 20.3 & 7.7 & -7.2 & 8 \\
\hline & & & pemetrexed or docetaxel $(n=174)$ & 22.8 & 3.0 & & \\
\hline NCT00777179[42] & NSCLC & Maintenance & vandetanib $(\mathrm{n}=75)$ & 15.6 & 2.7 & -6.2 & 7 \\
\hline & & & placebo $(n=42)$ & 20.8 & 1.7 & & \\
\hline Mok et al. 2017[84] & NSCLC & 2 & gefitinib + chemotherapy $(n=133)$ & 13.4 & 5.4 & -6.1 & 10 \\
\hline & & & placebo + chemotherapy $(n=132)$ & 19.5 & 5.4 & & \\
\hline Wu et al. 2014[53] & NSCLC & 1 & afatinib $(n=242)$ & 23.1 & 11.0 & -5.8 & 7 \\
\hline & & & gemcitabine + cislatin $(n=122)$ & 23.5 & 5.6 & & \\
\hline Hegewisch-Becker et al. & Colorectal & Maintenance & fluoropyrimidine + bevacizumab $(n=158)$ & 20.2 & 6.3 & -5.7 & 7 \\
\hline $2015[15]$ & & & no maintenance $(\mathrm{n}=158)$ & 23.1 & 3.5 & & \\
\hline Martin et al. 2015[32] & Breast & 1 & $\begin{array}{l}\text { letrozole or fulvestrant }+ \text { bevacizumab } \\
(\mathrm{n}=191)\end{array}$ & 52.1 & 19.3 & -5.6 & 6 \\
\hline & & & letrozole or fulvestrant $(n=189)$ & 52.8 & 14.4 & & \\
\hline Vincent et al. 2017[51] & Colorectal & 1 & erlotinib + capecitabine $(n=42)$ & 12.4 & 9.2 & -5.1 & 7 \\
\hline & & & capecitabine $(n=40)$ & 16.2 & 7.9 & & \\
\hline Herrlinger et al. 2016[27] & Glioblastoma & 1 & bevacizumb + irinotecan $(n=122)$ & 16.6 & 9.7 & -4.6 & 7 \\
\hline & & & temozolomide $(n=60)$ & 17.5 & 6.0 & & \\
\hline
\end{tabular}

\section{Conclusions}

There does not appear to be a difference in the magnitude of OS versus PFS benefits among biologic/targeted therapies versus their comparators. However, approximately $10 \%$ of comparisons showed a substantial ( $\geq 4$ month) difference in OS/PFS benefits versus non-biologic/targeted comparators. Among those, slightly over half $(n=16,64 \%)$ showed a benefit in OS versus the comparator, suggesting that the phenomenon of long OS and short PFS or vice versa may not be simply an artifact or a reflection of suboptimal trial design, but could possibly be due to their specific mechanism of action.

Study quality did not appear to have any role in the findings of large differential between OS and PFS or in the direction of study results, but rather publication status. The low study quality of unpublished studies could be either due to the inability to publish studies of this quality, or due to the lack of sufficient information in the 
clincaltrials.gov record to provide information to populate the study score across all variables. Similarly, studies reporting no gains in OS outcomes were if anything potentially more likely to have imbalance in post-study therapies $(80 \%$ of reporting studies) than those with positive OS findings (imbalance in post-study therapies was $21.8 \%$ of reporting studies); however, any interpretation of the role of post-study treatment imbalances is very limited due to the low number of studies reporting these data.

In summary, this analysis has evaluated the observation of small PFS benefit with large OS benefit as well as small OS benefit with large PFS benefit, and no systematic evidence was identified that this was a result of poor study design, post-study treatment or specific to any other factor evaluated in this review. The biologic and targeted agents identified with these differentials are generally FDA approved and NCCN-recommended therapies for the diseases in which they are evaluated. The observed OS and PFS benefits of these agents are likely due to the unique mechanism of action of these drugs, which should be better understood as the use of these novel agents increases and further scientific evidence is generated.

\section{Supplementary Material}

Supplementary search strategies and table. http://www.jcancer.org/v10p3717s1.pdf

\section{Acknowledgements}

The authors would like to thank Ms. Katherine Klamer from Indiana University (Indianapolis, IN) and Judith Bell, BSc and Emily Moss, BA, from RTI Health Solutions (Manchester, UK) for their assistance with study eligibility review, data entry and data validation for this study. The authors would also like to acknowledge Eric Wen $\mathrm{Su}, \mathrm{PhD}$ for the development and execution of the i2E strategy.

\section{Funding}

Resources to support a portion of the dual eligibility review and data entry were provided by Eli Lilly and Company to RTI Health Solutions.

\section{Competing Interests}

The authors have declared that no competing interest exists.

\section{References}

1. USDHHS. Guidance for Industry: Clinical Trial Endpoints for the Approval of Cancer Drugs and Biologics. 2007.

2. Booth CM, Eisenhauer EA. Progression-free survival: meaningful or simply measurable? J Clin Oncol. 2012; 30: 1030-3.

3. Shukuya T, Mori K, Amann JM, Bertino EM, Otterson GA, Shields PG, et al. Relationship between Overall Survival and Response or Progression-Free Survival in Advanced Non-Small Cell Lung Cancer Patients Treated with Anti-PD-1/PD-L1 Antibodies. J Thorac Oncol. 2016; 11: 1927-39.
4. Harris SJ, Brown J, Lopez J, Yap TA. Immuno-oncology combinations: raising the tail of the survival curve. Cancer Biol Med. 2016; 13: 171-93.

5. Tap WD, Jones RL, Van Tine BA, Chmielowski B, Elias AD, Adkins D, et al. Olaratumab and doxorubicin versus doxorubicin alone for treatment of soft-tissue sarcoma: an open-label phase $1 \mathrm{~b}$ and randomised phase 2 trial. Lancet. 2016; 388: 488-97.

6. Zer A, Prince RM, Amir E, Abdul Razak A. Evolution of Randomized Trials in Advanced/Metastatic Soft Tissue Sarcoma: End Point Selection, Surrogacy, and Quality of Reporting. J Clin Oncol. 2016; 34: 1469-75.

7. Muenst S, Soysal SD, Tzankov A, Hoeller S. The PD-1/PD-L1 pathway: biological background and clinical relevance of an emerging treatment target in immunotherapy. Expert Opin Ther Targets. 2015; 19: 201-11.

8. Antoniou G, Lee ATJ, Huang PH, Jones RL. Olaratumab in soft tissue sarcoma - Current status and future perspectives. Eur J Cancer. 2018; 92: 33-9.

9. Schwartz LH, Seymour L, Litiere S, Ford R, Gwyther S, Mandrekar S, et al. RECIST 1.1 - Standardisation and disease-specific adaptations: Perspectives from the RECIST Working Group. Eur J Cancer. 2016; 62: 138-45.

10. de Morton NA. The PEDro scale is a valid measure of the methodological quality of clinical trials: a demographic study. Aust J Physiother. 2009; 55: 129-33.

11. Higgins JP, Altman DG, Gotzsche PC, Juni P, Moher D, Oxman AD, et al. The Cochrane Collaboration's tool for assessing risk of bias in randomised trials. BMJ. 2011; 343: d5928.

12. Bhogal SK, Teasell RW, Foley NC, Speechley MR. The PEDro scale provides a more comprehensive measure of methodological quality than the Jadad scale in stroke rehabilitation literature. J Clin Epidemiol. 2005; 58: 668-73.

13. Sanborn RE, Patel JD, Masters GA, Jayaram N, Stephens A, Guarino M, et al. A randomized, double-blind, phase 2 trial of platinum therapy plus etoposide with or without concurrent vandetanib (ZD6474) in patients with previously untreated extensive-stage small cell lung cancer: Hoosier Cancer Research Network LUN06-113. Cancer. 2017; 123: 303-11.

14. NCCN. NCCN Clinical Practice Guidelines in Oncology (NCCN Guidelines): Soft Tissue Sarcoma ver 2.2019. J Natl Compr Canc Netw. 2019.

15. Hegewisch-Becker S, Graeven U, Lerchenmuller CA, Killing B, Depenbusch R, Steffens $\mathrm{C}-\mathrm{C}$, et al. Maintenance strategies after first-line oxaliplatin plus fluoropyrimidine plus bevacizumab for patients with metastatic colorectal cancer (AIO 0207): a randomised, non-inferiority, open-label, phase 3 trial. Lancet Oncol. 2015; 16: 1355-69.

16. Kawaguchi T, Ando M, Asami K, Okano Y, Fukuda M, Nakagawa H, et al. Randomized phase III trial of erlotinib versus docetaxel as second- or third-line therapy in patients with advanced non-small-cell lung cancer: Docetaxel and Erlotinib Lung Cancer Trial (DELTA). Journal of Clinical Oncology. 2014; 32: 1902-8.

17. Tabernero J, Yoshino T, Cohn AL, Obermannova R, Bodoky G, Garcia-Carbonero R, et al. Ramucirumab versus placebo in combination with second-line FOLFIRI in patients with metastatic colorectal carcinoma that progressed during or after first-line therapy with bevacizumab, oxaliplatin, and a fluoropyrimidine (RAISE): a randomised, double-blind, multicentre, phase 3 study.[Erratum appears in Lancet Oncol. 2015 Jun;16(6):e262; PMID: 26065608]. Lancet Oncol. 2015; 16: 499-508.

18. Beer TM, Kwon ED, Drake CG, Fizazi K, Logothetis C, Gravis G, et al. Randomized, Double-Blind, Phase III Trial of Ipilimumab Versus Placebo in Asymptomatic or Minimally Symptomatic Patients With Metastatic Chemotherapy-Naive Castration-Resistant Prostate Cancer. Journal of Clinical Oncology. 2017; 35: 40-7.

19. Belani CP, Yamamoto N, Bondarenko IM, Poltoratskiy A, Novello S, Tang J, et al. Randomized phase II study of pemetrexed/cisplatin with or without axitinib for non-squamous non-small-cell lung cancer. BMC Cancer. 2014; 14: 290.

20. Blumenschein GR, Jr., Smit EF, Planchard D, Kim DW, Cadranel J, De Pas T, et al. A randomized phase II study of the MEK1/MEK2 inhibitor trametinib (GSK1120212) compared with docetaxel in KRAS-mutant advanced non-small-cell lung cancer (NSCLC)dagger. Ann Oncol. 2015; 26: 894-901.

21. Burtness B, Powell M, Catalano P, Berlin J, Liles DK, Chapman AE, et al. Randomized Phase II Trial of Irinotecan/Docetaxel or Irinotecan/Docetaxel Plus Cetuximab for Metastatic Pancreatic Cancer: An Eastern Cooperative Oncology Group Study. Am J Clin Oncol. 2016; 39: 340-5.

22. Chauffert B, Feuvret L, Bonnetain F, Taillandier L, Frappaz D, Taillia H, et al. Randomized phase II trial of irinotecan and bevacizumab as neo-adjuvant and adjuvant to temozolomide-based chemoradiation compared with temozolomide-chemoradiation for unresectable glioblastoma: final results of the TEMAVIR study from ANOCEF+. Annals of Oncology. 2014; 25: 1442-7.

23. Doebele RC, Spigel D, Tehfe M, Thomas S, Reck M, Verma S, et al. Phase 2, randomized, open-label study of ramucirumab in combination with first-line pemetrexed and platinum chemotherapy in patients with nonsquamous, advanced/metastatic non-small cell lung cancer. Cancer. 2015; 121: 883-92.

24. Evans TRJ, Van Cutsem E, Moore MJ, Bazin IS, Rosemurgy A, Bodoky G, et al. Phase 2 placebo-controlled, double-blind trial of dasatinib added to gemcitabine for patients with locally-advanced pancreatic cancer. Annals of Oncology. 2017; 28: 354-61.

25. Gilbert MR, Dignam JJ, Armstrong TS, Wefel JS, Blumenthal DT, Vogelbaum $\mathrm{MA}$, et al. A randomized trial of bevacizumab for newly diagnosed glioblastoma. New England Journal of Medicine. 2014; 370: 699-708.

26. Gridelli C, Novello S, Zilembo N, Luciani A, Favaretto AG, De Marinis F, et al. Phase II randomized study of vandetanib plus gemcitabine or gemcitabine 
plus placebo as first-line treatment of advanced non-small-cell lung cancer in elderly patients. Journal of Thoracic Oncology: Official Publication of the International Association for the Study of Lung Cancer. 2014; 9: 733-7.

27. Herrlinger U, Schafer N, Steinbach JP, Weyerbrock A, Hau P, Goldbrunner R, et al. Bevacizumab Plus Irinotecan Versus Temozolomide in Newly Diagnosed O6-Methylguanine-DNA Methyltransferase Nonmethylated Glioblastoma: The Randomized GLARIUS Trial. Journal of Clinical Oncology. 2016; 34: $1611-9$

28. Kim YS, Cho EK, Woo HS, Hong J, Ahn HK, Park I, et al. Randomized Phase II Study of Pemetrexed Versus Gefitinib in Previously Treated Patients with Advanced Non-small Cell Lung Cancer. Cancer Res. 2016; 48: 80-7.

29. Leone F, Marino D, Cereda S, Filippi R, Belli C, Spadi R, et al. Panitumumab in combination with gemcitabine and oxaliplatin does not prolong survival in wild-type KRAS advanced biliary tract cancer: A randomized phase 2 trial (Vecti-BIL study). Cancer. 2016; 122: 574-81.

30. Li N, Ou W, Yang H, Liu Q-W, Zhang S-L, Wang B-X, et al. A randomized phase 2 trial of erlotinib versus pemetrexed as second-line therapy in the treatment of patients with advanced EGFR wild-type and EGFR FISH-positive lung adenocarcinoma. Cancer. 2014; 120: 1379-86.

31. Malka D, Cervera P, Foulon S, Trarbach T, de la Fouchardiere C, Boucher E, et al. Gemcitabine and oxaliplatin with or without cetuximab in advanced biliary-tract cancer (BINGO): a randomised, open-label, non-comparative phase 2 trial. Lancet Oncol. 2014; 15: 819-28.

32. Martin M, Loibl S, von Minckwitz G, Morales S, Martinez N, Guerrero A, et al. Phase III trial evaluating the addition of bevacizumab to endocrine therapy as first-line treatment for advanced breast cancer: the letrozole/fulvestrant and avastin (LEA) study. Journal of Clinical Oncology. 2015; 33: 1045-52.

33. Masi G, Salvatore L, Boni L, Loupakis F, Cremolini C, Fornaro L, et al. Continuation or reintroduction of bevacizumab beyond progression to first-line therapy in metastatic colorectal cancer: final results of the randomized BEBYP trial. Annals of Oncology. 2015; 26: 724-30.

34. McDermott DF, Sosman JA, Gonzalez R, Hodi FS, Linette GP, Richards J, et al. Double-blind randomized phase II study of the combination of sorafenib and dacarbazine in patients with advanced melanoma: a report from the 11715 Study Group. J Clin Oncol. 2008; 26: 2178-85

35. Middleton G, Palmer DH, Greenhalf W, Ghaneh P, Jackson R, Cox T, et al. Vandetanib plus gemcitabine versus placebo plus gemcitabine in locally advanced or metastatic pancreatic carcinoma (ViP): a prospective, randomised, double-blind, multicentre phase 2 trial. Lancet Oncol. 2017; 18: 486-99.

36. Miller VA, Hirsh V, Cadranel J, Chen YM, Park K, Kim SW, et al. Afatinib versus placebo for patients with advanced, metastatic non-small-cell lung cancer after failure of erlotinib, gefitinib, or both, and one or two lines of chemotherapy (LUX-Lung 1): a phase $2 b / 3$ randomised trial. Lancet Oncol. 2012; 13: 528-38.

37. Moore M, Gill S, Asmis T, Berry S, Burkes R, Zbuk K, et al. Randomized phase II study of modified FOLFOX-6 in combination with ramucirumab or icrucumab as second-line therapy in patients with metastatic colorectal cancer after disease progression on first-line irinotecan-based therapy. Annals of Oncology. 2016; 27: 2216-24.

38. Clinicaltrials.gov. Carboplatin and Paclitaxel With or Without Sorafenib Tosylate in Treating Patients With Stage III or Stage IV Melanoma That Cannot Be Removed by Surgery. 2015.

39. Clinicaltrials.gov. Study Of Sunitinib In Combination With Docetaxel Vs Docetaxel In Patients With Advanced Breast Cancer (SUN 1064). 2012.

40. Clinicaltrials.gov. A Study of Bevacizumab in Previously Untreated Extensive-Stage Small Cell Lung Cancer (SALUTE). 2011

41. Clinicaltrials.gov. Docetaxel in Combination With Zactima (ZD6474) in Patients With Locally Advanced Squamous Cell Carcinoma of the the Head and Neck. 2013

42. Clinicaltrials.gov. Phase II of Zactima Maintenance for Locally Advanced or Metastatic Non-small-cell Lung Carcinoma (NSCLC) Following Platinum-doublet Chemotherapy. 2016.

43. Clinicaltrials.gov. Phase III Trial Comparing Capecitabine in Combination With Sorafenib or Placebo in the Treatment of Locally Advanced or Metastatic HER2-Negative Breast Cancer. 2017.

44. Oza AM, Cook AD, Pfisterer J, Embleton A, Ledermann JA, Pujade-Lauraine E, et al. Standard chemotherapy with or without bevacizumab for women with newly diagnosed ovarian cancer (ICON7): overall survival results of a phase 3 randomised trial. Lancet Oncol. 2015; 16: 928-36.

45. Paz-Ares LG, Biesma B, Heigener D, von Pawel J, Eisen T, Bennouna J, et al. Phase III, randomized, double-blind, placebo-controlled trial of gemcitabine/cisplatin alone or with sorafenib for the first-line treatment of advanced, nonsquamous non-small-cell lung cancer. J Clin Oncol. 2012; 30: 3084-92.

46. Ramlau R, Gorbunova V, Ciuleanu TE, Novello S, Ozguroglu M, Goksel T, et al. Aflibercept and Docetaxel versus Docetaxel alone after platinum failure in patients with advanced or metastatic non-small-cell lung cancer: a randomized, controlled phase III trial. J Clin Oncol. 2012; 30: 3640-7.

47. Schuler M, Yang JC, Park K, Kim JH, Bennouna J, Chen YM, et al. Afatinib beyond progression in patients with non-small-cell lung cancer following chemotherapy, erlotinib/gefitinib and afatinib: phase III randomized LUX-Lung 5 trial. Ann Oncol. 2016; 27: 417-23.
48. Shaw AT, Kim DW, Nakagawa K, Seto T, Crino L, Ahn MJ, et al. Crizotinib versus chemotherapy in advanced ALK-positive lung cancer. N Engl J Med. 2013; 368: 2385-94.

49. Shen L, Li J, Xu J, Pan H, Dai G, Qin S, et al. Bevacizumab plus capecitabine and cisplatin in Chinese patients with inoperable locally advanced or metastatic gastric or gastroesophageal junction cancer: randomized, double-blind, phase III study (AVATAR study). Gastric Cancer. 2015; 18: $168-76$

50. Vergote IB, Jimeno A, Joly F, Katsaros D, Coens C, Despierre E, et al. Randomized phase III study of erlotinib versus observation in patients with no evidence of disease progression after first-line platin-based chemotherapy for ovarian carcinoma: a European Organisation for Research and Treatment of Cancer-Gynaecological Cancer Group, and Gynecologic Cancer Intergroup study. Journal of Clinical Oncology. 2014; 32: 320-6.

51. Vincent MD, Breadner D, Soulieres D, Kerr IG, Sanatani M, Kocha W, et al. Phase II trial of capecitabine plus erlotinib versus capecitabine alone in patients with advanced colorectal cancer. Fut Oncol. 2017; 13: 777-86.

52. Wirth LJ, Dakhil S, Kornek G, Axelrod R, Adkins D, Pant S, et al. PARTNER: An open-label, randomized, phase 2 study of docetaxel/cisplatin chemotherapy with or without panitumumab as first-line treatment for recurrent or metastatic squamous cell carcinoma of the head and neck. Oral Oncol. 2016; 61: 31-40.

53. Wu YL, Zhou C, Hu CP, Feng J, Lu S, Huang Y, et al. Afatinib versus cisplatin plus gemcitabine for first-line treatment of Asian patients with advanced non-small-cell lung cancer harbouring EGFR mutations (LUX-Lung 6): an open-label, randomised phase 3 trial. Lancet Oncol. 2014; 15: 213-22.

54. Allen JW, Moon J, Redman M, Gadgeel SM, Kelly K, Mack PC, et al. Southwest Oncology Group S0802: a randomized, phase II trial of weekly topotecan with and without ziv-aflibercept in patients with platinum-treated small-cell lung cancer. J Clin Oncol. 2014; 32: 2463-70.

55. Belani $\mathrm{CP}$, Dahlberg SE, Rudin $\mathrm{CM}$, Fleisher $\mathrm{M}$, Chen $\mathrm{HX}$, Takebe $\mathrm{N}$, et al. Vismodegib or cixutumumab in combination with standard chemotherapy for patients with extensive-stage small cell lung cancer: A trial of the ECOG-ACRIN Cancer Research Group (E1508). Cancer. 2016; 122: 2371-8.

56. Bokemeyer C, Bondarenko I, Makhson A, Hartmann JT, Aparicio J, de Braud $\mathrm{F}$, et al. Fluorouracil, leucovorin, and oxaliplatin with and without cetuximab in the first-line treatment of metastatic colorectal cancer. J Clin Oncol. 2009; 27: 663-71.

57. Borghaei H, Paz-Ares L, Horn L, Spigel DR, Steins M, Ready NE, et al. Nivolumab versus Docetaxel in Advanced Nonsquamous Non-Small-Cell Lung Cancer. New England Journal of Medicine. 2015; 373: 1627-39.

58. Buikhuisen WA, Scharpfenecker M, Griffioen AW, Korse CM, van Tinteren H, Baas P. A Randomized Phase II Study Adding Axitinib to Pemetrexed-Cisplatin in Patients with Malignant Pleural Mesothelioma: A Single-Center Trial Combining Clinical and Translational Outcomes. Journal of Thoracic Oncology: Official Publication of the International Association for the Study of Lung Cancer. 2016; 11: 758-68.

59. Govindan R, Szczesna A, Ahn MJ, Schneider CP, Gonzalez Mella PF, Barlesi F, et al. Phase III Trial of Ipilimumab Combined With Paclitaxel and Carboplatin in Advanced Squamous Non-Small-Cell Lung Cancer. Journal of Clinical Oncology. 2017; 35: 3449-57.

60. Clinicaltrials.gov. Phase 3 Trial in Squamous Non Small Cell Lung Cancer Subjects Comparing Ipilimumab Versus Placebo in Addition to Paclitaxel and Carboplatin. 2018.

61. Han $B$, Jin $B, C$ hu $T$, Niu $Y$, Dong $Y, X u$ J et al Combination of chemotherapy and gefitinib as first-line treatment for patients with advanced lung adenocarcinoma and sensitive EGFR mutations: A randomized controlled trial. Int J Cancer. 2017; 141: 1249-56.

62. Herbst RS, Baas P, Kim DW, Felip E, Perez-Gracia JL, Han JY, et al. Pembrolizumab versus docetaxel for previously treated, PD-L1-positive, advanced non-small-cell lung cancer (KEYNOTE-010): a randomised controlled trial. Lancet. 2016; 387: 1540-50.

63. Jonasch E, Hasanov E, Corn PG, Moss T, Shaw KR, Stovall S, et al. A randomized phase 2 study of MK-2206 versus everolimus in refractory renal cell carcinoma. Annals of Oncology. 2017; 28: 804-8.

64. Lee DH, Lee JS, Kim SW, Rodrigues-Pereira J, Han B, Song XQ, et al. Three-arm randomised controlled phase 2 study comparing pemetrexed and erlotinib to either pemetrexed or erlotinib alone as second-line treatment for never-smokers with non-squamous non-small cell lung cancer. Eur J Cancer. 2013; 49: 3111-21.

65. Lee SM, Lewanski CR, Counsell N, Ottensmeier C, Bates A, Patel N, et al. Randomized trial of erlotinib plus whole-brain radiotherapy for NSCLC patients with multiple brain metastases. J Natl Cancer Inst. 2014; 106.

66. Clinicaltrials.gov. A Study of Capecitabine (Xeloda) and Bevacizumab as a First-line Therapy in Patients With Metastatic Colorectal Cancer. 2016.

67. Clinicaltrials.gov. THOR Study: A Study of Continued Herceptin (Trastuzumab) in Combination With Second Line Chemotherapy in Patients With HER2 Positive Metastatic Breast Cancer. 2014.

68. Clinicaltrials gov. An Efficacy and Safety Study With Vandetanib to Treat Inoperable or Relapsed Malignant Mesothelioma. 2016.

69. Clinicaltrials.gov. A Randomized Phase II Study of Cisplatin and Etoposide in Combination With Either Hedgehog Inhibitor GDC-0449 or IGF-1R MOAB IMC-A12 for Patients With Extensive Stage. 2018. 
70. Clinicaltrials.gov. An Efficacy Study in Gastric and Gastroesophageal Junction Cancer Comparing Ipilimumab Versus Standard of Care Immediately Following First Line Chemotherapy. 2016.

71. Pirker R, Pereira JR, Szczesna A, von Pawel J, Krzakowski M, Ramlau R, et al. Cetuximab plus chemotherapy in patients with advanced non-small-cell lung cancer (FLEX): an open-label randomised phase III trial. Lancet. 2009; 373: 1525-31.

72. Powles T, Huddart RA, Elliott T, Sarker SJ, Ackerman C, Jones R, et al. Phase III, Double-Blind, Randomized Trial That Compared Maintenance Lapatinib Versus Placebo After First-Line Chemotherapy in Patients With Human Epidermal Growth Factor Receptor 1/2-Positive Metastatic Bladder Cancer. Journal of Clinical Oncology. 2017; 35: 48-55.

73. Propper D, Davidenko I, Bridgewater J, Kupcinskas L, Fittipaldo A, Hillenbach $\mathrm{C}$, et al. Phase II, randomized, biomarker identification trial (MARK) for erlotinib in patients with advanced pancreatic carcinoma. Annals of Oncology. 2014; 25: 1384-90.

74. Scagliotti G, Novello S, von Pawel J, Reck M, Pereira JR, Thomas M, et al. Phase III study of carboplatin and paclitaxel alone or with sorafenib in advanced non-small-cell lung cancer. J Clin Oncol. 2010; 28: 1835-42.

75. Vilgrain V, Pereira H, Assenat E, Guiu B, Ilonca AD, Pageaux GP, et al. Efficacy and safety of selective internal radiotherapy with yttrium-90 resin microspheres compared with sorafenib in locally advanced and inoperable hepatocellular carcinoma (SARAH): an open-label randomised controlled phase 3 trial. Lancet Oncol. 2017; 18: 1624-36.

76. Yoon HH, Bendell JC, Braiteh FS, Firdaus I, Philip PA, Cohn AL, et al. Ramucirumab combined with FOLFOX as front-line therapy for advanced esophageal, gastroesophageal junction, or gastric adenocarcinoma: a randomized, double-blind, multicenter Phase II trial. Annals of Oncology. 2016; 27: 2196-203.

77. Wu YL, Zhou C, Liam CK, Wu G, Liu X, Zhong Z, et al. First-line erlotinib versus gemcitabine/cisplatin in patients with advanced EGFR mutation-positive non-small-cell lung cancer: analyses from the phase III, randomized, open-label, ENSURE study. Ann Oncol. 2015; 26: 1883-9.

78. Chinot OL, Wick W, Mason W, Henriksson R, Saran F, Nishikawa R, et al. Bevacizumab plus radiotherapy-temozolomide for newly diagnosed glioblastoma. N Engl J Med. 2014; 370: 709-22.

79. Schwartzberg LS, Franco SX, Florance A, O'Rourke L, Maltzman J, Johnston S. Lapatinib plus letrozole as first-line therapy for HER-2+ hormone receptor-positive metastatic breast cancer. Oncologist. 2010; 15: 122-9.

80. Krop IE, Kim SB, Martin AG, LoRusso PM, Ferrero JM, Badovinac-Crnjevic T, et al. Trastuzumab emtansine versus treatment of physician's choice in patients with previously treated HER2-positive metastatic breast cancer (TH3RESA): final overall survival results from a randomised open-label phase 3 trial. Lancet Oncol. 2017; 18: 743-54.

81. Guan $\mathrm{Z}, \mathrm{Xu}$ B, DeSilvio ML, Shen $\mathrm{Z}$, Arpornwirat $\mathrm{W}$, Tong $\mathrm{Z}$, et al. Randomized trial of lapatinib versus placebo added to paclitaxel in the treatment of human epidermal growth factor receptor 2-overexpressing metastatic breast cancer. J Clin Oncol. 2013; 31: 1947-53.

82. Furtado M, Johnson R, Kruger A, Turner D, Rule S. Addition of bortezomib to standard dose chop chemotherapy improves response and survival in relapsed mantle cell lymphoma. Br J Haematol. 2015; 168: 55-62.

83. Harrington K, Berrier A, Robinson M, Remenar E, Housset M, de Mendoza $\mathrm{FH}$, et al. Randomised Phase II study of oral lapatinib combined with chemoradiotherapy in patients with advanced squamous cell carcinoma of the head and neck: rationale for future randomised trials in human papilloma virus-negative disease. Eur J Cancer. 2013; 49: 1609-18.

84. Mok TSK, Kim SW, Wu YL, Nakagawa K, Yang JJ, Ahn MJ, et al. Gefitinib Plus Chemotherapy Versus Chemotherapy in Epidermal Growth Factor Receptor Mutation-Positive Non-Small-Cell Lung Cancer Resistant to First-Line Gefitinib (IMPRESS): Overall Survival and Biomarker Analyses. J Clin Oncol. 2017; 35: 4027-34. 